\title{
A cost analysis of options for schistosomiasis control MDA programs targeting children aged five years and below in uMkhanyakude District of KwaZulu-Natal Province, South Africa
}

Mhlengi Vella Ncube ( $\square$ mhlengivncube@yahoo.com )

University of KwaZulu-Natal School of Nursing and Public Health https://orcid.org/0000-0002-8317-9856

Moses John Chimbari

University of KwaZulu-Natal School of Nursing and Public Health

Research

Keywords: NA

Posted Date: June 22nd, 2020

DOI: https://doi.org/10.21203/rs.3.rs-33856/v1

License: (c) (i) This work is licensed under a Creative Commons Attribution 4.0 International License. Read Full License 


\section{Abstract}

\section{Background}

Schistosomiasis negatively impacts early childhood development. Inclusion of children aged five years and below in mass drug administration (MDA) programs for controlling schistosomiasis could improve early childhood development in communities where the disease is endemic. We estimated the projected cost of implementing a schistosomiasis control MDA program for children aged five years and below in the uMkhanyakude district of South Africa.

\section{Method}

We calculated the cost of implementing a schistosomiasis MDA program targeting children aged five years and below using an economies of scaled based cost function. We further compared different labor composition simulations to determine the most affordable and available human resources to implement the program. We also explored programs to which the MDA program could be integrated; and estimated what the costs for would be. Moreover, we simulated costeffectiveness and determined the cost drivers for each simulation considered.

\section{Results}

A ward-based outreach team (WBOT) for implementing a schistosomiasis MDA program targeting children 5 years old and below was the best labor composition option. The simulations conducted indicated that treating children in batches of 2500 using the WBOT team approach could reduce the cost of treatment by $53 \%$ compared to treating the children on batches of 500. Integrating a schistosomiasis MDA targeting children aged 5 years and below with the immunization program was estimated to cost $3 \%$ less than integration with the deworming and Vitamin A supplementation program indicating that the former option is more cost-effective. Praziquantel, the drug that is used to treat schistosomiasis contributed $59 \%$ of the total cost for such a program.

\section{Conclusion.}

We estimated that US\$4.3 million would be needed to implement a cost effective MDA program targeting children 5 years old and blow over 3 years in uMkhanyakude district.

\section{Contributions To The Literature}

- This study showed how economies of scale and economies of scope impact the cost of implementing schistosomiasis control mass drug administration programs. We also found that it is more cost-effective to integrate a schistosomiasis control mass drug administration program targeting children aged five years and below with a mobile clinic based programs than with the clinic based immunisation program. We showed that the cost of branded praziquantel is a major cost driver of implementing a schistosomiasis control MDA program where generic forms of praziquantel are prohibited.

\section{Introduction}

Schistosomiasis remains prevalent in many countries especially in those economically disadvantaged[1, 2]. Globally, several initiatives and programs have been implemented to control the disease but the debate on the costing of such programs remain inconclusive $[1,3]$. In a quest to promote health for all, the United Nations General Assembly adopted Agenda 2030 with seventeen Sustainable Development Goals (SDGs) in September 2015. Goal three of the SDGs seeks to promote health for all people of all ages[4]. Scholars and practitioners have since identified several diseases that need to be targeted to fulfil SDG3; one of them schistosomiasis which is widely prevalent in economically disadvantaged communities[5]. The cost of schistosomiasis treatment can be prohibitive to the affected communities[6]., contributes 
significantly to the accessibility of schistosomiasis control interventions to the community [7]. Schistosomiasis and the other neglected tropical diseases (NTDs) require funds for control and prevention programs in order to reach the target populations $[8,9]$.

The source of funding for schistosomiasis control is often external to the communities that are affected. Most of the funds for the controlling schistosomiasis and the other neglected tropical diseases (NTDs) comes from the governments of the United States of America and the United Kingdom[10]. A significant portion of funds for NTDs is provided by philanthropic organizations such as the Bill and Melinda Gates Foundation, END FUND[10] and the Schistosomiasis Control Initiative (SCI). Further support for schistosomiasis control has been provided by the pharmaceutical industry through a WHO initiative to mobilize the donation of praziquantel (PZQ). PQZ is the only WHO approved drug for treating schistosomiasis. The cost of schistosomiasis control can be significant but varies by region. For instance, in Egypt it cost US\$10 million annually[11] and the country successfully controlled the disease using domestic funds[11]. Although philanthropic organizations have taken a keen interest in financing of schistosomiasis, the World Bank recommends local financing as the best option for sustainable control of schistosomiasis[12].

Much emphasis has been placed on Schistosomiasis treatment as not treating infected children negatively impacts early childhood development. This is of great concern to multilateral organizations such as the World Bank which recommends countries to invest in early childhood development programs for poverty alleviation and diminishing the probability of acquiring disease at an early age[13]. One such investment is the PZQ mass drug administration for schistosomiasis as preventive treatment in children aged five years and below[14]. Schistosomiasis is endemic in six provinces of (Limpopo, North West, Mpumalanga, Eastern Cape, KwaZulu-Natal, Gauteng) South Africa. In the uMkhanyakude district of KwaZulu-Natal and other economically disadvantaged communities, schistosomiasis is widespread and as such, children below the age of five need schistosomiasis preventive treatment $[14,15]$.

Community-wide treatment has been found to be the most cost-effective strategy to control schistosomiasis[16]. To align schistosomiasis control chemotherapy with the WHO strategy for global schistosomiasis control, the inclusion of children aged five years and below is paramount $[17,18]$. The actual cost for schistosomiasis control mass drug administration (MDA) programs is difficult to infer from literature because of the variation in methods and cost lines used in the studies. Furthermore, it is only in 2014 that a working group to provide recommendations on the inclusion of children aged five years and below in schistosomiasis control preventive chemotherapy programs as part of the Integrated Management of Childhood Illnesses (IMCl)[19]. Thus, there is dearth in the discourse of implementing a schistosomiasis control MDA for children aged 5 years and below.

The South African government, through the National Department of Health, plans to implement a nationwide schistosomiasis control MDA program to control and eliminate the disease [20]. To support this government initiative, we therefore sought to carry out an analysis of the cost of implementing a schistosomiasis control MDA program for children five years old and below. We conducted the study in uMkhanyakude district, KwaZulu-Natal province where the disease is widespread. The uMkhanyakude district has five sub-districts. Each of the sub-districts has an average of 15000 children aged five years and below[21]. Our analysis considered the economies of scale and economies of scope associated with the mass treatment program. This paper, therefore, is intended to inform policymakers on the budgetary requirements for designing and implementing a schistosomiasis control MDA program for children aged five years and below in uMkhanyakude district.

\section{Methods}

\section{Site selection and study design}


uMkhanyakude district was purposively selected because it is one of the districts where schistosomiasis is endemic in South Africa[22, 23]. The child health program in uMkhanyakude district is implemented through the district Department of Health[24]. The cost analysis was done from a government perspective to establish the cost that the healthcare system in the district would bear in implementing a schistosomiasis control MDA program for children aged five years and below. The data was collected between August and November 2019. All South African rand (ZAR) values were converted to United States Dollars (US\$) for ease of reference and international comparability. In this regard, the data point exchange rate as at 30 November 2019 was US\$1:ZAR14.1 [25, 26].

\section{Cost Classifications}

The costs of implementing the schistosomiasis program were classified into two broad categories; fixed and variable costs. Fixed costs are those that remain constant despite changes in the number of children treated in the MDA program. Variable costs on the other hand, change with the number of children treated in the MDA program. The cost of clinic infrastructure was treated as sunk costs and hence were regarded as irrelevant for decision-making. Cost of training was also excluded from the costing exercise mainly because training for the MDA program would be done alongside IMCI training thus making it an indirect cost. Consequently, only direct costs to the treatment program were included in the costing exercise for this study. Table 1 shows the classification of costs for the MDA program.

Table 1

Classification of costs for the schistosomiasis control MDA programs

\begin{tabular}{|c|c|c|c|}
\hline Fixed Costs & Source & Variable costs & Source \\
\hline Computers & online sources & Food & personal communication \\
\hline Laboratory Tests & $\begin{array}{l}\text { National Health Laboratory Services } \\
\text { (NHLS) }\end{array}$ & Dose Syringe & online sources \\
\hline Labour & $\begin{array}{l}\text { Department of Public Service } \\
\text { Administration }\end{array}$ & $\begin{array}{l}\text { Office } \\
\text { resources }\end{array}$ & online sources \\
\hline $\begin{array}{l}\text { Mobile clinic (running } \\
\text { costs) }\end{array}$ & Right2Care[27] & $\begin{array}{l}\text { Praziquantel } \\
(P Z Q)\end{array}$ & $\begin{array}{l}\text { uMkhanyakude Department of } \\
\text { Health }\end{array}$ \\
\hline Pestle and Mortar & online sources & & \\
\hline Weight scales & online sources & & \\
\hline
\end{tabular}

\section{Data Analysis}

All calculations were done on Microsoft Excel Spreadsheet. The average cost of treatment per child was calculated by dividing the sum of the fixed cost and the variable costs (aggregate) by the total number of children treated.

Subsequently, differential costing was determined by subtracting the average cost per child or total cost of treatment in a specific treatment of scenario from an alternative scenario.

Economies of scale were calculated by determining the difference between the average cost of treating children when the only variable in the treatment scenario is the number of children treated. Economies of scope were calculated by dividing the fixed costs with the number of programs that shared resources; adding the variable costs that were specific to the number of children treated in the MDA program and comparing that to a scenario where the MDA program was not integrated with any other program.

The total cost was calculated based on the number of children where the economies of scale were highest. Costeffectiveness was calculated based on the average cost of treating an additional child to achieve the $75 \%$ treatment coverage that is recommended by the $\mathrm{WHO}[28,29]$. The cost proportions were calculated by comparing the contributions of each cost line to the total cost of treating $75 \%$ of the children aged five years and below in uMkhanyakude district. We 
used the cost of branded praziquantel in our cost analysis, therefore, cost sensitivity analysis was calculated based on the cost of using generic praziquantel and on a scenario that praziquantel was donated to the country. We used generic praziquantel as the recommended treatment to lower the cost of schistosomiasis treatment in South Africa. The donated praziquantel was used in the sensitivity determination because WHO is encouraging pharmaceutical companies to donate praziquantel to communities that need to implement schistosomiasis control mass drug administration programs[19].

\section{Ethical Clearance}

Ethical clearance was granted by the University of KwaZulu-Natal Biomedical Research Ethics Committee (reference number: BE403/18) and the KwaZulu-Natal Department of Health Research Committee (reference number NHRD_201809_007).

\section{Results}

\section{Resource Planning and Economies of scale}

Community healthcare programs in uMkhanyakude are often implemented or supported by ward-based outreach teams (WBOTs)[24]. A WBOT consists of a professional nurse, a staff nurse and community care givers (CCGs)[24]. If the simulation treatment team consists of the ward-based outreach team (WBOT) and one pediatric specialist, the average cost, per child, of treating 500 children in a schistosomiasis control MDA program for children aged below five years in uMkhanyakude District would be estimated to be US\$60. If the model treatment team consists of a Medical Officer and WBOT the estimated average cost per child treating 500 children drops to US\$57 indicating a decline of $5 \%$. However, a WBOT only model treatment team requires US\$27 which is $20 \%$ less per child compared to when a pediatrician is part of the treatment team. In the simulation, if the number of children treated increased to 2500 , the average cost per child reduced to US\$30 (51\% drop) when a pediatrician is part of the treatment team; reduced by $49 \%$ when a medical officer is part of the treatment team instead of a pediatrician; and by $53 \%$ when the treatment team is the WBOT. If the number of children is increased to 18500 , the average cost of treatment per child reduces by $42 \%$ from US\$60 to US\$23, by $62 \%$ for a pediatrician led team, by $52 \%$ for a Medical Officer led team and $53 \%$ for a WBOT only treatment team. Table 2 shows the project's simulated economies of scale.

Table 2

Program's economies of scale analysis and resource planning.

\begin{tabular}{|c|c|c|c|c|}
\hline & Paediatric Specialist and WBOT & Medical Officer and WBOT. & WBOT only & \\
\hline 500 children & $0 \%$ & $-5 \%$ & $-20 \%$ & \\
\hline 2500 children & $-51 \%$ & $-49 \%$ & $-53 \%$ & \\
\hline 13500 children & $-62 \%$ & $-52 \%$ & $-53 \%$ & \\
\hline \multicolumn{5}{|c|}{ Descriptive Statistics } \\
\hline & Max (US\$) & Max Scenario & Min (US\$) & Min Scenario \\
\hline 500 children & 60 & Paediatric Specialist and WBOT & 48 & WBOT only \\
\hline 2500 children & 30 & Paediatric Specialist and WBOT & 22 & WBOT only \\
\hline 13500 children & 23 & Paediatric Specialist and WBOT & 17 & WBOT only \\
\hline
\end{tabular}

If only the WBOT is used to treat 2500 children, and laboratory diagnosis is limited to a sample of 500 children, the average cost of treatment per child in the MDA program is $18 \%$ lower than when all the children are screened and treated for schistosomiasis. If 18500 children are treated by a WBOT based on results of testing 500 samples, the cost of the 
MDA program would be $26 \%$ lower than when all the children are tested. A scenario in which only a sample of 500 children was tested for schistosomiasis using laboratory methods was used for further calculations. Figure 1 depicts economies of scale for each treatment model.

As shown in Fig. 1, the average cost per child in a schistosomiasis control MDA program decreases sharply when the number of children treated are increased from less than 500 children to about 2500 children. A gradual decrease in the cost of treatment per child was observed if the number of children to be treated was increased to more than 2500.

\section{Implementation strategy and economies of scope.}

uMkhanyakude district has fixed clinics and School Health Mobile Clinics where the vaccination program is done. In comparing the cost of using the fixed clinics and mobile clinics, we assumed that four additional community caregivers would be required in the mobile clinics to make up for the absence of the parents at the schools when the School Health Mobile Clinic is used in crèches and early childhood development centers. It cost $14 \%$ more per child to treat the children in the School Health Mobile Clinic than it would cost in the fixed clinics when 500 children were treated. If 2500 children are treated, it costs $6 \%$ more to treat children in the School Health Mobile Clinic compared to the fixed clinic. Figure 2 shows the economies of scope for the program based on simulations.

Currently, the vaccination program in uMkhanyakude District is done in the fixed clinics while the school health mobile clinics are used for the deworming program. The deworming program is integrated with the vitamin A supplementation program. If the schistosomiasis control MDA is in integrated with the vaccination program, it costs $33 \%$ less to treat 500 than it would to treat 500 children if the MDA is implemented independently in a fixed clinic. This cost difference reduces to $14 \%$ when 2500 are treated. If the schistosomiasis control program is integrated with the deworming and vitamin $\mathrm{A}$ supplementation program, the cost of treating 500 children becomes $47 \%$ lower than treating the children in an independent schistosomiasis control MDA program using a mobile clinic. The cost difference reduces to $22 \%$ when 2500 children are treated.

If the schistosomiasis control MDA for children aged five years and below is integrated to the fixed clinic, it costs $9 \%$ more to treat 500 children than when the MDA program is integrated with the deworming and vitamin A supplementation program. The cost difference reduced to $3 \%$ when 2500 were treated. There was no difference in the cost of using either of the options to treat at 17000 or more.

\section{Total Costs and Cost Effectiveness Analysis}

Based on the WHO recommended coverage of $75 \%$, the study found that the estimated total cost of implementing the MDA is US\$1.087 million when the schistosomiasis control MDA program for children aged five years and below is integrated with the immunization program. Integrating the schistosomiasis control MDA program for children aged five years and below with the deworming and vitamin A supplementation programs was US\$1.056 million indicating a 3\% reduction in costs from the former.

At present, coverage for the immunization program in uMkhanyakude district is $85 \%$ while that of the deworming and Vitamin A program is 74\%[21]. The cost effectiveness is calculated based on the average cost per additional child above or to reach the $75 \%$ treatment coverage recommended by $\mathrm{WHO}[28,29]$. If the schistosomiasis control MDA program is integrated with the immunization program, 75\% coverage would require US\$100 per additional child to reach that level. When the MDA program is integrated with the deworming and vitamin A supplementation program, the additional cost per child is US\$1,246 to reach the $75 \%$ coverage. Assuming that deworming and vitamin A supplementation program reaches the $85 \%$ coverage similar to that of the immunization program, the additional cost per child to achieve the WHO coverage of $75 \%$ would be US\$97. Table 2 shows the total cost and cost effectiveness analysis of the different options for treatment programs. 


\section{Cost Ratios}

PZQ was the main cost driver of the MDA program and accounted for $59 \%$ of the costs of the schistosomiasis control MDA program for children aged five years and below when either the fixed clinics or mobile clinics were used. For both the fixed clinics and the mobile clinics, dose syringes contributed $26 \%$ of the costs, food contributed $13 \%$ while labor and laboratory costs contributed $1 \%$ each to the total costs of the schistosomiasis control MDA program as shown in Figs. 3 below.

\section{Sensitivity Analysis}

The main cost driver of the treatment program was the cost of PZQ. The sensitivity of the total costs to the use of generic drugs is detailed in Table 3 :

Table 3

Sensitivity Analysis

\begin{tabular}{|lllll|}
\hline Percentage difference in cost of PZQ (brand to Generic) & $39 \%$ & $\mathbf{4 5 \%}$ & $\mathbf{6 0 \%}$ & $\mathbf{1 0 0 \%}$ \\
\hline Percentage difference in total cost of treatment & $-23 \%$ & $-27 \%$ & $-35 \%$ & $-59 \%$ \\
\hline
\end{tabular}

If the average cost of generic PZQ is less than the branded PZQ, the total cost of the schistosomiasis control MDA program for children aged five years and below would reduce by $23 \%$. When the cost of generic drugs is less than the branded drug by $45 \%$, there is a $27 \%$ reduction in the cost of the MDA program. A $60 \%$ reduction in the generic $P Z Q$ compared to the branded drug gave a $35 \%$ reduction in the total cost of the MDA program. In the case of donated PZQ the treatment program costs $59 \%$ less that when branded PZQ is used to treat schistosomiasis in an MDA program targeting children aged five years and below.

\section{Discussion}

Resource planning

The Essential Medicines List of South Africa stipulates that a pediatric specialist should be involved in the treatment with praziquantel of children aged below five years[30]. South Africa is currently experiencing a shortage of specialist skills across all fields of medicine[31]. Rural areas such as uMkhanyakude District are the most affected by the shortage of medical professionals such as pediatric specialists[32]. General practitioners are also inadequate to service the public health needs of South Africa's rural districts including the uMkhanyakude district. As a result, many of the healthcare programs are managed by WBOTs[24]. The inclusion of participation of a pediatrician in the schistosomiasis control MDA program for children aged five years and below was found to be the most expensive option in the human resources planning choices that are available. Treatment done using a WBOT only is the most affordable and practical option for a schistosomiasis control MDA program for children aged five years and below.

\section{Economies of scale.}

The cost of treating children aged 5 years and below with PZQ in a schistosomiasis control MDA program decreases with an increase in the number of children treated. This decrease in costs is a consequence of economies of scale[33, 34]. Economies of scale occur because the fixed programmatic costs such as computers, weight scales and labor remain constant even when the number of children increases[33,35]. The economies of scale increase exponentially until the increasing variable costs are significant enough to buffer the reduction in the average cost of treatment per child that occurs as result of the fixed costs. uMkhanyakude district has five sub-districts. Each of the sub-districts has an average of 15000 children that are aged five years and below. Our findings indicate that economies of scale will exist when the MDA program is implemented at sub-district level (population: 15 000). In practice, the economies of scale will only exist 
up to a specific threshold beyond which diseconomies of scale will cause the average of cost of treating a child in the MDA program will increase[16, 36].

Diseconomies of scale occur when the increase in variable costs due to an increase in the number of children being treated outweighs the reduction in the average cost per child that results from the fixed costs remaining constant when the number of children that are treated increases[34]. When diseconomies of scale are experienced operational strategies to reduce costs reduce upscaling costs[34]. A point at which economies of scale reduce significantly was identified as 2500 children receiving treatment under the program. We recommend that each schistosomiasis control treatment station in uMkhanyakude district should target about 2500 children to be cost effective.

\section{Economies of scope}

The WHO recommends that schistosomiasis control MDA be integrated with existing child health activities[37]. Healthcare program integration improves the financial sustainability and operational efficiency that is needed by NTDs control programs[38]. In uMkhanyakude district, the immunization program and the deworming and vitamin $\mathrm{A}$ supplementation programs could be used to implement the schistosomiasis control MDA program for children aged five years and below[24]. When programs are integrated, some of the common costs are shared between more than one program, thus creating economies of scope[34]. Resource planning is essential in the exploitation of economies of scope because the economies of scope will increase when the biggest cost drivers of individual programs are the same in the programs that are being integrated[39].

\section{Cost drivers}

The biggest cost driver of the MDA program was the cost of praziquantel, which contributed $59 \%$ of the total cost of the MDA program in the model used in this study. In South Africa, only branded PZQ is licensed for use. The cost of PZQ in South Africa is 50 times more that the WHO expected cost[40]. The licensing of generic PZQ could make a significant contribution to the control of schistosomiasis in South Africa and in lowering the cost of schistosomiasis control MDA programs in the country. In Canada the use of generics reduces the cost of drugs by an average of $88 \%[41]$. In South Africa, the difference in cost of drugs (brand to generic) was found to average $39 \% ; 45 \%$ and $60 \%$ for the high cost; medium cost and low cost drugs respectively[42]. We used these parameters in our sensitivity analysis and found that the use of generic PZQ could lower the total cost of the MDA program by between $23 \%-35 \%$. Donated PZQ could reduce the cost of a schistosomiasis control MDA program for children aged five years old and below in the uMkhanyakude district by about $59 \%$.

The second largest cost driver was the dose syringes. The dose syringes could be replaced by spoons provided strict infection prevention control protocols are followed. The use of spoons has been successful in clinical studies[18]. Food also contributed significantly to the cost of the MDA program. Food is important in the MDA program to improve the systemic assimilation of PZQ and to ameliorate some of the side effects of PZQ. Schistosomiasis affects poor communities; hence it is expected that most of the children affected by schistosomiasis will have inadequate access to food. Food is therefore an integral part of the treatment process. In instances where feeding schemes are part of the crèches and early childhood development programs, $13 \%$ of the total cost of treatment could be removed by treating the children immediately after mealtime. Cost driver reduction measures are more effective when they add value to an already cost-effective program.

\section{Total cost and Cost-effectiveness analysis}

The cost effectiveness of PZQ MDA programs in the control of schistosomiasis is understood and well document[43]. We considered programmatic cost-effectiveness in our study to determine the best programmatic strategy that could be used to implement a schistosomiasis control MDA program for children aged five years and below in uMkhanyakude. Based on 
the existing coverage (85\%) of the fixed clinic immunization[21], it is estimated that US\$100 per child would be required to achieve the $\mathrm{WHO}$ recommended $75 \%$ coverage when the MDA program is integrated with the immunization program. When the MDA program is integrated with the mobile clinic deworming and vitamin A supplementation whose coverage is $74 \%$, US $\$ 1246$ would be required to close the $1 \%$ coverage gap towards the WHO recommended coverage. Based on the cost per additional child only, the immunization program is a more cost-effective approach. However, on assumption that the coverage of the MDA programs is the same at $85 \%$ the deworming and vitamin A supplementation program is a more cost-effective vehicle for the schistosomiasis control MDA program for children aged five years and below. The deworming and Vitamin A supplementation program from 46\% in 2017 to $74 \%$ in 2019[21, 44]. This increase in coverage shows that economic determination of program integration choices requires predictive analysis of program performance when costs are projected. The use of cost functions that could resolve economies of scale and economies of scope illustrates the complexity of the economic evaluations that are required to inform program integration. In addition to economic evaluations, policy and political decisions may influence the decision making process.

\section{Strengths and weaknesses of the study}

The strengths of this study is premised on the fact that it took into account the economies of scale and economies of scope to determine the cost of implementing a schistosomiasis control MDA program for children aged five years and below. The study therefore aligns to the programmatic decision making process that is involved in implementing PZQ MDA programs. In addition, the processes that govern healthcare interventions in South Africa is similar throughout the country making the study applicable to several districts in South Africa where schistosomiasis is endemic. The findings of this study also account for the recommended improvement in schistosomiasis treatment coverage to $85 \%[1]$. The limitation of the study is that some of the initial costs such as community healthcare worker training were not considered in this study as this information was not readily available.

\section{Conclusion}

The planned schistosomiasis control MDA program for school going children in South Africa could be expanded to include children aged five years and below in the districts where schistosomiasis is endemic. Implementing a schistosomiasis control MDA program for children aged five years and below in uMkhanyakude district can be done by a WBOT. Such an MDA program could be integrated with either the immunization program or the deworming and Vitamin A supplementation program. Using a discount rate of 5\%, a 3-year budget for a schistosomiasis control MDA program for children aged five years and below in uMkhanyakude would require US\$4. 3 million in the next three years. Cost cutting measures for the implementation of the MDA program could include using generic or donated PZQ.

\section{Abbreviations}

CCG

Community Care Giver

$\mathrm{DoH}$

The South Africa Department of Health

ECD

Early Childhood Development

MDA

Mass Drug Administration

NHLS

National Health Laboratory Services

NTD 
Neglected Tropical Diseases

PZQ

Praziquantel

$\mathrm{SCl}$

Schistosomiasis Control Initiative

SDG

Strategic Development Goal

STH

Soil Transmitted Helminths

TB

Tuberculosis

UKZN

University of KwaZulu-Natal

WBOT

Ward Based Outreach Teams

WHO

World Health Organisation

ZAR

South African Rand

\section{Declarations}

\section{Acknowledgements}

The authors would like to acknowledge Prof Mabutho Sibanda for assisting in reviewing this manuscript. We are grateful to the uMkhanyakude Health District for supporting our research activities.

\section{Availability of data and materials}

Data will be made available upon request from the corresponding author.

\section{Funding}

University of KwaZulu-Natal.

NIHR Global Health Research Unit Tackling Infections To Benefit Africa

British Academy's Early Childhood Development Program.

The above funders contributed to the financing of the materials and travelling that was required to carry out the study only. The views expressed in this publication are those of the author(s) and not necessarily those of the NHS, the National Institute of Health Research, or the Department of Health.

\section{Authors' contributions}

MVN conceived the study in collaboration with MC. Both authors were involved in data analysis and interpretation of results. Both authors revised and approved the final manuscript. 


\section{Consent for publication}

Not applicable.

\section{Competing interests}

The authors declare no that they have no competing interests.

\section{Ethics approval and consent to participate}

Ethical clearance was obtained from the University of KwaZulu-Natal Biomedical Research Ethics Committee (reference number: BE403/18) and the KwaZulu-Natal Department of Health Research Committee (reference number NHRD_201809_007).

\section{Author details}

${ }^{1}$ School of Nursing and Public Health, College of Health Sciences, University of KwaZulu- Natal, Durban, South Africa

\section{References}

1. Li EY, et al. Improving public health control of schistosomiasis with a modified WHO strategy: a model-based comparison study. The Lancet Global Health. 2019;7(10):e1414-22.

2. Person B, et al., Community knowledge, perceptions, and practices associated with urogenital schistosomiasis among school-aged children in Zanzibar, United Republic of Tanzania. PLoS neglected tropical diseases, 2016. 10(7).

3. Salari P, et al. Cost of interventions to control schistosomiasis: A systematic review of the literature. PLOS Neglected Tropical Diseases. 2020;14(3):e0008098.

4. Fernandez RM. SDG3 Good Health and Well-Being: Integration and Connection with Other SDGs. Good Health and Well-Being, 2020: p. 629-636.

5. De Neve J-W, et al. Health, financial, and education gains of investing in preventive chemotherapy for schistosomiasis, soil-transmitted helminthiases, and lymphatic filariasis in Madagascar: A modeling study. PLoS Negl Trop Dis. 2018;12(12):e0007002-2.

6. Sacolo-Gwebu H, Kabuyaya M, Chimbari M, Knowledge, attitudes and practices on schistosomiasis and soiltransmitted helminths among caregivers in Ingwavuma area in uMkhanyakude district, South Africa. BMC Infectious Diseases, 2019. 19(1): p. 734.

7. Danso-Appiah A, et al. Health seeking behaviour and utilization of health facilities for schistosomiasis-related symptoms in ghana. PLoS Negl Trop Dis. 2010;4(11):e867-7.

8. Reed SL, McKerrow JH. Why Funding for Neglected Tropical Diseases Should Be a Global Priority. Clin Infect Dis. 2018;67(3):323-6.

9. Nonvignon J, et al., The role of health systems in the control of neglected tropical diseases in Sub-Saharan Africa, in Neglected Tropical Diseases-Sub-Saharan Africa. 2016, Springer. p. 385-405.

10. Hotez PJ, et al. World neglected tropical diseases day. PLOS Neglected Tropical Diseases. 2020;14(1):e0007999.

11. WHO. Egypt leverages domestic funding to eliminate schistosomiasis.. 2016 [cited 2018 March 2018]; Available from: http://www.who.int/neglected_diseases/news.Egypt_leverages_domestic_funding_to_eliminate_schistosomiasis/en/.

12. WHO, Investing to Overcome the Global Impact of Neglected Tropical Diseases: Third WHO Report on Neglected Tropical Diseases 2015, P.P. Holmes, editor. 2015, WHO. 
13. Sayre RK, et al., Investing in early childhood development: review of the World Bank's recent experience. 2015: The World Bank.

14. Stothard JR, et al. Schistosomiasis in African infants and preschool children: let them now be treated! Trends in Parasitology. 2013;29(4):197-205.

15. Kabuyaya M, Chimbari MJ, Mukaratirwa S. Efficacy of praziquantel treatment regimens in pre-school and school aged children infected with schistosomiasis in sub-Saharan Africa: a systematic review. Infectious diseases of poverty. 2018;7(1):73.

16. Turner $\mathrm{HC}$, et al. Evaluating the variation in the projected benefit of community-wide mass treatment for schistosomiasis: Implications for future economic evaluations. Parasites Vectors. 2017;10:213.

17. WHO. WHO. Schistosomiaisis Control and preventive chemotherapy. 2018 [cited 2018 July 2018]; Available from: http://www.who.int/schistosomiasis/strategy/en/.

\section{Figures}

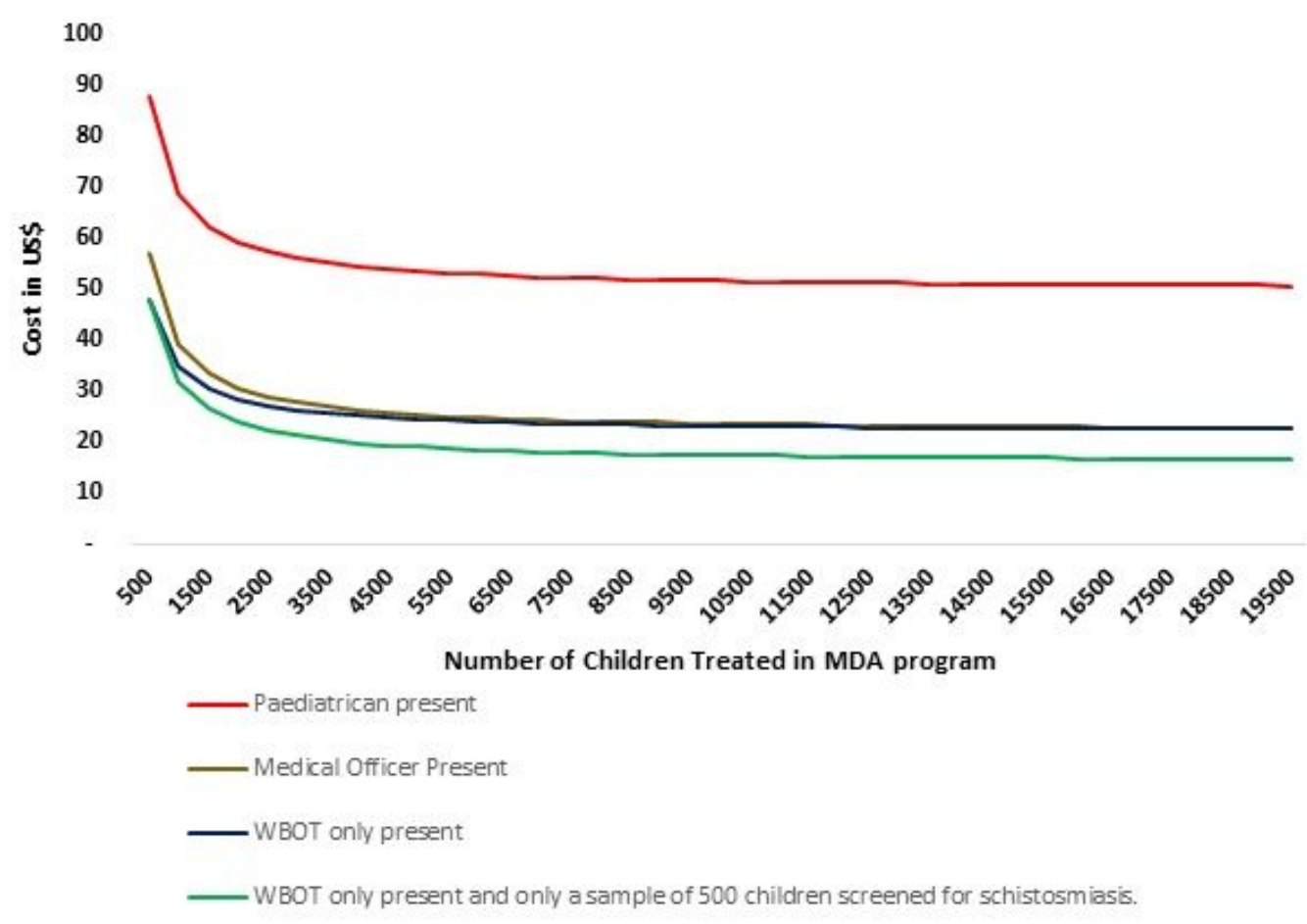

\section{Figure 1}

Economies of scale per treatment model 


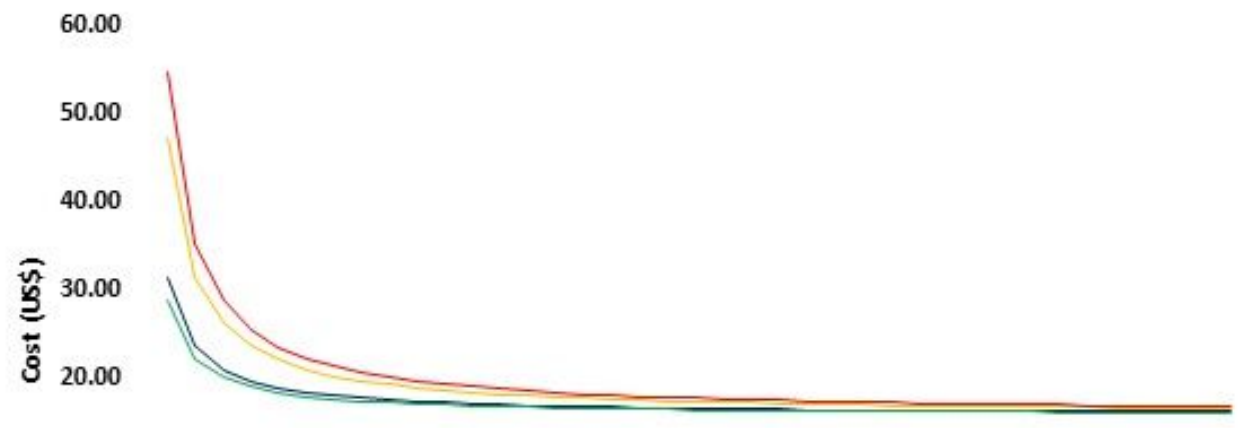

10.00

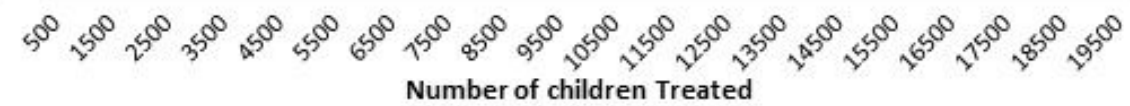

$$
\begin{aligned}
& \text { Fixed Clinic } \quad \text { School Health Mobile Clinic _ A B }
\end{aligned}
$$

Figure 2

Economies of Scope of the treatment program

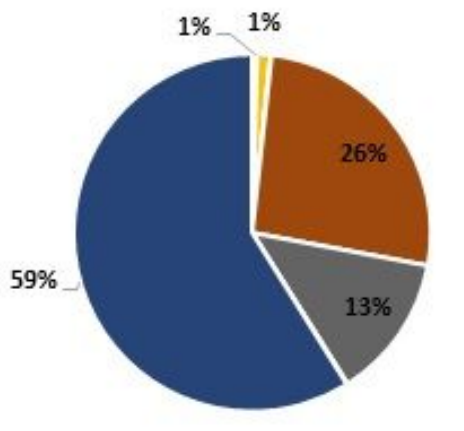

$$
\begin{array}{ll}
\text { - Mobile clinic } & \text { | Computers } \\
\text { - Mobile clinic } & \text { | Pestle and Mortar } \text { | Weight scales } \\
\text { | Food } & \text { | Office supplies } \quad \text { - Praziquantel }
\end{array}
$$

\section{Figure 3}

Cost ratios for mobile clinic 


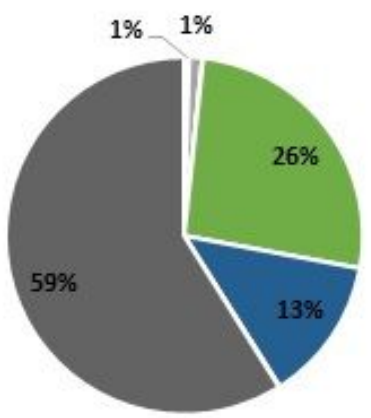

$$
\begin{aligned}
& \text { - Computers } \quad \text { - Laboratory Tests }=\text { Labour } \\
& \text { = Pestle and Mortar } \mid \text { Weight scales | Dose syringe } \\
& \text { - Food | Office supplies = Praziquantel }
\end{aligned}
$$

\section{Figure 4}

Cost ratios for fixed clinic use

\section{Supplementary Files}

This is a list of supplementary files associated with this preprint. Click to download.

- IMPSD2000317RebuttalandCorrectionsv2.docx

- IMPSD2000317RebuttalandCorrectionsv1.0.docx

- IMPSD2000317CommentsandRebuttalTable.docx

- MhlengicoverletterBMCImplementationScience.docx 